\title{
Regiospecific Synthesis of New Non-Condensed Heteropolycyclic Systems from $\beta$-Heteroaryl $\beta$-methoxyvinyl Trihalomethyl Ketones
}

\author{
Helio G. Bonacorso*, Marlí R. Oliveira, Michelle B. Costa, Letícia B. da Silva, Nilo Zanatta, \\ Marcos A. P. Martins and Alex F. C. Flores
}

Departamento de Química,Universidade Federal de Santa Maria, 97105-900 Santa Maria - RS, Brazil

\begin{abstract}
Reações de ciclo-condensação envolvendo 1,1,1-trifluor(cloro)-4-metoxi-4-(2-heteroaril)-3buten-2-onas ( $\beta$-alcoxivinil trialometil cetonas) e furoil-, tenoil- e isonicotinoil-hidrazinas (heteroarilhidrazidas), realizadas sob condições brandas e em meio alcoólico, forneceram em passo reacional único e regioespecificamente, uma série inédita composta por doze heteroaroil-2-pirazolinas trialometil heteroaril substituídas em rendimentos de 50-78\%; um novo sistema heteropolicíclico não condensado.
\end{abstract}

\begin{abstract}
A novel series of twelve heteroaroyl-2-pyrazolines trihalomethyl and substituted heteroaryl, as non-condensed heteropolycyclic systems, have been synthesized in one-step in 50-78\% yield from the regiospecific cyclocondensation reaction of 1,1,1-trifluoro(chloro)-4-methoxy-4-(2-furyl)- and 4-(2-thienyl)-3-buten-2-ones ( $\beta$-alkoxyvinyl trihalometil ketones) with furoic hydrazide, 2 thiophenecarboxylic hydrazide and isonicotinic acid hydrazide (heteroaroylhydrazines) under mild conditions in methanol as solvent.
\end{abstract}

Keywords: 2-pyrazolines, pyrazoles, 4-alkoxy-1,1,1-trihalo-alk-3-en-2-ones, hydrazides, heteroaroylhydrazines, hydrazines

\section{Introduction}

Although many methods have been published for the synthesis of $1 \mathrm{H}$-pyrazoles and their derivatives, attempts to perform the synthesis of a simple 4,5-dihydro- $1 H$-pyrazoles (2-pyrazolines), has not yet been sucessfully achieved. ${ }^{1-3}$ By conventional procedure, pyrazoles have been obtained by directly reacting $\beta$-diketones with hydrazine. ${ }^{1}$ However, in most cases, 5 -hydroxy-4,5-dihydro- $1 H$-pyrazoles have been obtained when the $\mathrm{N}-1$ atom is substituted with a strong electron-withdrawing group which stabilizes the $-\mathrm{OH}$ group and possibly hinders the elimination of the water molecule and the subsequent aromatization of the pyrazoline ring. ${ }^{1}$

Regarding pharmacological applications, 2-pyrazolines (4,5-dihydro- $1 \mathrm{H}$-pyrazoles) have been found to possess antitumor, ${ }^{4}$ antibacterial, antifungal, antiviral, antiparasitic, antitubercular and insecticidal

* e-mail: heliogb@base.ufsm.br and others properties. ${ }^{5-16}$ Some of these compounds also presented anti-inflammatory, anti-diabetic, anesthetic and analgesic properties. ${ }^{13-15}$ For example, in a recent work Mamolo et al. ${ }^{16}$ have synthesized a series of 5-aryl-1-isonicotinoyl-3-(pyridin-2-yl)-4,5-dihydro$1 H$-pyrazoles in a three-step in low yields, which involved an aldol condensation, cyclocondensation with hydrazine and $\mathrm{N}$-acylation with isonicotinoyl chloride. These 2-pyrazolines showed an interesting antimycobacterial activity in vitro.

In 2002, our research group reported ${ }^{17}$ a new approach for the synthesis of a series of four ketones as 1,1,1trifluoro(chloro)-4-methoxy-4-(2-furyl)- and 4-(2thienyl)-3-buten-2-ones and their 4,4,4-trihalo-1-(2heteroaryl)-1,3-butanediones derivatives. The autors also showed that mixtures of $\beta$-alkoxyvinyl trifluoromethyl ketones and the respective 1,3butanediones derived from 2-acetylthiophene and 2acetylfuran react with hydroxylamine hydrochloride, dry hydrazine and thiosemicarbazide hydrochloride leading to 3-(2-thienyl)- and 3-(2-furyl)azoles 
derivatives. In this case, only one isoxazoline and one 2-pyrazoline have been isolated. In particular, the synthetic potential of $\beta$-alkoxyvinyl trihalomethyl ketones to obtain series of novel heterocycles of five, ${ }^{18}$ six, ${ }^{19}$ seven membered rings ${ }^{20}$ and more recently bisheterocyles ${ }^{21}$ has been reported by us.

Considering the biological importance of 2-pyrazolines and the fact that trichloromethylated analogs, such as, bisheteroaryl ketones are not yet known, it would be nice to demonstrate a new synthetic application of $\beta$-heteroaryl- $\beta$-methoxyvinyl trihalomethyl ketones. ${ }^{17}$ Herein, the synthesis and isolation of a novel series of twelve heteroaroyl pyrazolines trihalomethyl and heteroaryl substituted, a heteropolycyclic system, from the reaction of 1,1,1trifluoro(chloro)-4-methoxy-4-(2-furyl)- and 4-(2thienyl)-3-buten-2-ones with furoic hydrazide, 2thiophenecarboxylic hydrazide and isonicotinic acid hydrazide, is presented (Scheme 1).

\section{Results and Discussion}

The reaction of 1,1,1-trifluoro(chloro)-4-methoxy-4-(2furyl) and 4-(2-thienyl)-3-buten-2-ones (1a-b, 2a-b) with furoic hydrazide, 2-thiophenecarboxylic hydrazide and isonicotinic acid hydrazide was carried out in a 1:1 molar ratio in anhydrous methanol and all reactions were monitored by TLC. The most satisfactory results were obtained when the reactions were performed under mild conditions at room temperature for 24 hours. It was observed that the reactions performed at higher temperatures resulted in polymerization and lower yields. After the addition of the reagents, the contents darkened and formation of brown and gray solids occurred. After 24 hours, the contents were cooled to $10^{\circ} \mathrm{C}$ and the solid products were isolated by filtration. The crude crystals were recrystallized either from methanol or acetone. Under these conditions, the derivatives of these three series of hydrazides, viz., 3-(2-furyl)- or 3-(2-thienyl)-5-hydroxy5-trifluoro(chloro)methyl-4,5-dihydro- $1 H$-1 - (2furoyl)pyrazoles (3a-b, 4a-b), 3-(2-furyl)- or 3-(2-thienyl)5-hydroxy-5-trifluoro(chloro)methyl-4,5-dihydro- $1 \mathrm{H}-1$-(2thenoyl)pyrazoles (5a-b, 6a-b) and 3-(2-furyl)- or 3-(2thienyl)-5-hydroxy-5-trifluoro(chloro)methyl-4,5-dihydro$1 H$-1-(isonicotinoyl)pyrazoles (7a-b, 8a-b) were obtained regiospecifically in one-step and in satisfactory yields (64 $53 \%, 78-62 \%$ and $66-50 \%$ ), respectively. Our experiments demonstrated that the trihalomethyl and the heteroaroyl groups at the positions 5 and 1 of the 2-pyrazolines 3-8, respectively, act as protective groups with electron withdrawing effect, thus stabilizing the $-\mathrm{OH}$ group at $\mathrm{C}-5$ and possibly hindering the elimination of water and the subsequent aromatization of the five-membered ring. Due to the thermodynamic stability of 2-pyrazolines $\mathbf{3 - 8}$, the presence of a trihalomethyl group on the $\beta$-methoxyvinyl trihalomethyl ketones $\mathbf{1}$ and $\mathbf{2}$ and the heteroaroyl groups on the dinucleophiles (hydrazides) were the determining factor of the regiochemistry of the reaction. In addition, the heteroaryl substituent (2-thienyl and 2-furyl) attached at position $\mathbf{4}$ of the ketones $\mathbf{1}$ and $\mathbf{2}$ and the adopted synthetic method produced no observable effects on the regiochemistry of the cyclo-condensation reaction.

The best reaction conditions, selected physical and spectral data are presented in the experimental part. The 1,1,1-trifluoro(chloro)-4-methoxy-4-(2-furyl)- and

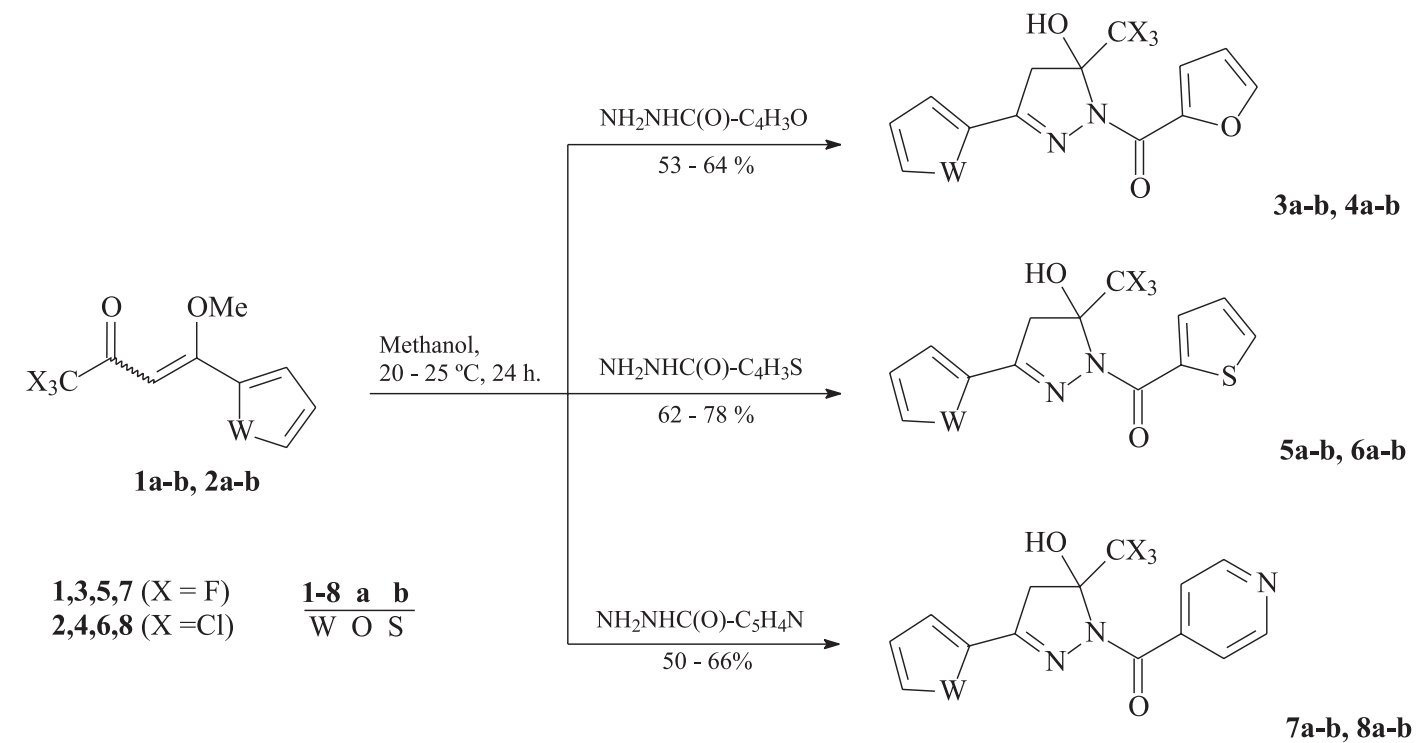

Scheme 1. 
4-(2-thienyl)-3-buten-2-ones 1a-b and 2a-b were prepared according to the procedure developed previously. ${ }^{17}$

The unambiguous ${ }^{1} \mathrm{H}$ and ${ }^{13} \mathrm{C}$ NMR chemical shift assignments of 3-aryl-5-hydroxy-5-trifluoro(chloro)methyl4,5-dihydro-1H-1-(heteroaroyl)pyrazoles (3a-b, 4a-b, 5a-b, 6a-b 7a-b and 8a-b), were made with the help of homo- and heteronuclear COSY, HMQC and HMBC 2D NMR periments and by comparison with NMR data of others 2- pyrazolines previously synthesised in our laboratory. Compounds 3-8 show the ${ }^{1} \mathrm{H}$ NMR chemical shifts of the diastereotopic methylene protons ( $\mathrm{H} 4 \mathrm{a}$ and $\mathrm{H} 4 \mathrm{~b}$ ) as a characteristic $\mathrm{AB}$ system and as a doublet in the range of $\delta 3.90$ to 4.12 and another doublet in the range of $\delta 3.53$ to 3.85 , respectively, with a geminal coupling constant in the range of ${ }^{2} J=18.8-$ 19.4 Hz. The trifluoromethylated heterocycles 3a-b, 5a-b and $7 \mathbf{a}-\mathbf{b}$ present the typical ${ }^{13} \mathrm{C}$ chemical shifts of pyrazoline ring carbons at $\delta 147.4$ (C3), 44.1 (C4), 92.1 (C5), 123.4 $\left(\mathrm{CF}_{3}\right)$. The trichloromethylated compounds $\mathbf{4 a - b}, \mathbf{6 a - b}$ and 8a-b present the typical ${ }^{13} \mathrm{C}$ chemical shifts of pyrazoline ring carbons at $\delta 148.4$ (C3), 46.7 (C4), 102.7 (C5), 103.1 $\left(\mathrm{CCl}_{3}\right)$. The carbonyl carbon for the trifluoro(chloro)methylated series 3-6 (furoyl and thenoyl derivatives) shows signals in the range of $\delta 151.1$ to 160.6. As expected, for isonicotinoyl derivatives (7a-b, 8a-b) the carbonyl carbon shows signals in the range of $\delta 164.8$ to 165.4 .

\section{Conclusions}

We have developed an useful, simple and convenient procedure to obtain new trifluoro(chloro)methylated hydroxypyrazolines, as heteropolycycles derived from thiophene, furan and pyridine under mild conditions. Furthermore, we have been able to use for the first time bheteroaryl-b-methoxyvinyl trihalomethyl ketones $\mathbf{1}$ and $\mathbf{2}$ in cyclocondensation reactions with heteroaroylhydrazines.

\section{Experimental}

Unless otherwise indicated all common reagents and solvents used in the present work were obtained from commercial suppliers without further purification. All melting points were determined using open capillaries on a Electrothermal Mel-Temp 3.0 apparatus and are uncorrected. ${ }^{1} \mathrm{H}$ and ${ }^{13} \mathrm{C}$ NMR spectra were acquired on a Bruker DPX 200 spectrometer $\left({ }^{1} \mathrm{H}\right.$ at $200.13 \mathrm{MHz}$ and ${ }^{13} \mathrm{C}$ at $50.32 \mathrm{MHz}$ ), $5 \mathrm{~mm}$ sample tubes, $298 \mathrm{~K}$, digital resolution $\pm 0.01 \mathrm{ppm}$, in methyl sulfoxide- $d_{6}$ for $\mathbf{3 a - i}$ using TMS as internal reference. IR spectra were recorded on a Nicolet Magna 550 IR spectrometer. The CHN elemental analysis were performed on a Perkin-Elmer 2400 CHN elemental analyzer (São Paulo University, USP/ Brazil).

General procedure for the synthesis of 3-heteroaryl-5hydroxy-5-trifluoro(trichloro)methyl-4,5-dihydro-1H-1(heteroaroyl)pyrazoles (3-8)

To a stirred solution of 4-alkoxy-4-heteroaryl-1,1,1trifluoro(trichloro)-3-buten-2-one $\mathbf{1 a - b}$ or $\mathbf{2 a - b}$ (5 mmoles) in $6 \mathrm{~mL}$ of methanol, dry 2-thiophenecarboxylic hydrazide, furoic hydrazide or isonicotinic acid hydrazide ( 5 mmoles) was added at $20-25^{\circ} \mathrm{C}$. The mixture was stirred at room temperature $\left(20-25^{\circ} \mathrm{C}\right)$ for 24 hours. After cooling $\left(<10^{\circ} \mathrm{C}\right)$, the crystalline solids were isolated by filtration, washed with cold methanol and recrystallized from acetone.

Data for 3-(2-furyl)-5-hydroxy-5-trifluoromethyl-4,5dihydro-1H-1-(2-furoyl)pyrazole (3a)

Gray crystals (62\% yield), mp $166-168{ }^{\circ} \mathrm{C}$ (acetone). ${ }^{1} \mathrm{H}$ NMR (DMSO-d 6 ): (Pyrazole) $\delta 8.35$ (1H, s, OH); 3.90 (1H, d, J 18.7, H4a); 3.54 (1H, d, J 18.8, H4b). (Furoyl) $\delta$ 8.04 (1H, s, H5); 7.64 (1H, d, J3.0, H3); $6.78-6.73$ (1H, m, H4). (Furyl) $\delta 7.99$ (1H, s, H5); 7.20 (1H, d, J 3.2, H3); 6.78 - 6.73 (1H, m, H4). ${ }^{13} \mathrm{C}$ NMR (DMSO-d $)$ : (Pyrazole) $\delta$ 146.0 (C3); $124.6\left(\mathrm{CF}_{3}, J_{\mathrm{CF}} 283.8\right) ; 92.0$ (C5, $\left.{ }^{2} J 34.1\right) ; 43.5$ (C4). (Furoyl) $\delta 155.3$ (C=O); 146.8 (C2); 145.5 (C5); 120.3 (C4); 112.0 (C3). (Furyl) $\delta 145.2$ (C2); 144.0 (C5); 114.9 (C4); 112.3 (C3). IR (KBr) $v_{\max } / \mathrm{cm}^{-1}: 3329(\mathrm{OH}) ; 1644$ $(\mathrm{C}=\mathrm{O}) ; 1555,1468(\mathrm{C}=\mathrm{C})$. Anal. Calc. for $\mathrm{C}_{13} \mathrm{H}_{9} \mathrm{~F}_{3} \mathrm{~N}_{2} \mathrm{O}_{4}$ (314.22): C, 49.69; H, 2.89; N, 8.92\%. Found: C, 50.16; H, $2.84 ; \mathrm{N}, 8.95 \%$.

Data for 3-(2-thienyl)-5-hydroxy-5-trifluoromethyl-4,5dihydro-1H-1-(2-furoyl)pyrazole (3b)

Gray crystals ( $53 \%$ yield), mp $149-150{ }^{\circ} \mathrm{C}$ (acetone). ${ }^{1} \mathrm{H}$ NMR (DMSO-d $)$ ): (Pyrazole) $\delta 8.33$ (1H, s, OH); 3.97 (1H, d, $J$ 19.2, H4a); 3.62 (1H, d, J 19.0, H4b). (Furoyl) $\delta$ 8.01 (1H, s, H5); 7.62 (1H, d, J 2.8, H3); 6.75 (1H, dd, J 3.4, $J$ 1.6, H4). (Thienyl) $\delta 7.80(1 \mathrm{H}, \mathrm{d}, J 5.0, \mathrm{H} 5) ; 7.53(1 \mathrm{H}, \mathrm{d}$, J3.6, H3); 7.20 (1H, dd, J4.4, J4.2, H4). ${ }^{13} \mathrm{C} \mathrm{NMR}$ (DMSO$\left.\mathrm{d}_{6}\right)$ : (Pyrazole) $\delta 148.8(\mathrm{C} 3) ; 123.2\left(\mathrm{CF}_{3}, J_{\mathrm{CF}} 284.1\right) ; 92.5$ (C5, ${ }^{2} J$ 33.7); 44.3 (C4). (Furoyl) $\delta 151.1$ (C=O); 146.9 (C2); 145.5 (C5); 120.1 (C4); 112.1 (C3). (Thienyl) $\delta 133.1$ (C2); 131.3 (C3); 130.2 (C5); 128.3 (C4). IR (KBr) $v_{\text {max }} / \mathrm{cm}^{-1}: 3242(\mathrm{OH}) ; 1645(\mathrm{C}=\mathrm{O}) ; 1560,1470(\mathrm{C}=\mathrm{C})$. Anal. Calc. for $\mathrm{C}_{13} \mathrm{H}_{9} \mathrm{~F}_{3} \mathrm{~N}_{2} \mathrm{O}_{3} \mathrm{~S}$ (330.28): C, 47.28; H, 2.75; N, $8.48 \%$. Found: C, 47.53; H, 2.70; N, 8.58\%. 
Data for 3-(2-furyl)-5-hydroxy-5-trichloromethyl-4,5dihydro-1H-1-(2-furoyl)pyrazole (4a)

Gray crystals (61\% yield), mp $162-164{ }^{\circ} \mathrm{C}$ (acetone). ${ }^{1} \mathrm{H}_{\mathrm{H}} \mathrm{MR}$ (DMSO-d $\mathrm{d}_{6}$ ): (Pyrazole) $\delta 8.25(1 \mathrm{H}, \mathrm{s}, \mathrm{OH}) ; 3.97$ (1H, d, $J$ 19.4, H4a); 3.70 (1H, d, $J$ 19.2, H4b). (Furoyl) $\delta$ $8.02(1 \mathrm{H}, \mathrm{s}, \mathrm{H} 5) ; 7.57(1 \mathrm{H}, \mathrm{d}, J 3.4, \mathrm{H} 3) ; 6.76-6.71(1 \mathrm{H}, \mathrm{m}$, H4). (Furyl) $\delta 7.97(1 \mathrm{H}, \mathrm{d}, J$ 1.2, $\mathrm{H} 5) ; 7.24(1 \mathrm{H}, \mathrm{d}, J 3.2$, H3); $6.76-6.71(1 \mathrm{H}, \mathrm{m}, \mathrm{H} 4) .{ }^{13} \mathrm{C}$ NMR (DMSO-d $)_{6}$ : (Pyrazole) $\delta 146.2(\mathrm{C} 3) ; 103.1\left(\mathrm{CCl}_{3}\right) ; 102.5$ (C5); 46.1 (C4). (Furoyl) $\delta 157.2(\mathrm{C}=\mathrm{O}) ; 146.9(\mathrm{C} 2) ; 145.5(\mathrm{C} 5) ; 120.7$ (C4); 112.1 (C3). (Furyl) $\delta 145.6$ (C2); 144.8 (C5); 115.3 (C4); $112.3(\mathrm{C} 3)$. IR (KBr) $v_{\text {max }} / \mathrm{cm}^{-1}: 3288(\mathrm{OH}) ; 1639$ $(\mathrm{C}=\mathrm{O}) ; 1554,1470(\mathrm{C}=\mathrm{C})$. Anal. Calc. for $\mathrm{C}_{13} \mathrm{H}_{9} \mathrm{Cl}_{3} \mathrm{~N}_{2} \mathrm{O}_{4}$ (363.58): C, 42.95; H, 2.50; N, 7.70\%. Found: C, 43.17; H, $2.40 ; \mathrm{N}, 7.65 \%$.

Data for 3-(2-thienyl)-5-hydroxy-5-trichloromethyl-4,5dihydro-1H-1-(2-furoyl)pyrazole (4b)

Gray crystals (64\% yield), mp $139-141{ }^{\circ} \mathrm{C}$ (acetone). ${ }^{1} \mathrm{H}$ NMR (DMSO-d $\mathrm{d}_{6}$ ): (Pyrazole) $\delta 8.25$ (1H, s, OH); 4.09 (1H, d, $J$ 19.0, H4a); 3.80 (1H, d, $J$ 19.2, H4b). (Furoyl) $\delta$ $8.02(1 \mathrm{H}, \mathrm{s}, \mathrm{H} 5) ; 7.68$ (1H, d, J 2.4, H3); 6.76 (1H, dd, J 3.4, $J$ 1.8, H4). (Thienyl) $\delta 7.81$ (1H, d, J 4.2, H5); 7.50 (1H, d, $J 3.2, \mathrm{H} 3) ; 7.21(1 \mathrm{H}, \mathrm{dd}, J 4.8, J 3.6, \mathrm{H} 4) .{ }^{13} \mathrm{C} \mathrm{NMR}$ (DMSO$\left.\mathrm{d}_{6}\right)$ : (Pyrazole) $\delta 150.4$ (C3); $103.2\left(\mathrm{CCl}_{3}\right) ; 103.1$ (C5); 46.9 (C4). (Furoyl) $\delta 157.0$ (C:O); 146.9 (C2); 145.5 (C5); 120.4 (C4); 112.1 (C3). (Thienyl) $\delta 132.8$ (C2); 131.4 (C3); 130.4 (C5); $128.3(\mathrm{C} 4)$. IR (KBr) $v_{\max } / \mathrm{cm}^{-1}: 3145(\mathrm{OH})$; $1629(\mathrm{C}=\mathrm{O})$; 1550, $1461(\mathrm{C}=\mathrm{C})$. Anal. Calc. for $\mathrm{C}_{13} \mathrm{H}_{9} \mathrm{Cl}_{3} \mathrm{~N}_{2} \mathrm{O}_{3} \mathrm{~S}$ (379.65): C, 41.13; H, 2.39; N, 7.38\%. Found: C,41.41; H, 2.44; N, 7.38\%.

Data for 3-(2-furyl)-5-hydroxy-5-trifluoromethyl-4,5dihydro-1H-1-(2-thenoyl)pyrazole (5a)

Gray crystals (67\% yield), mp $150-152{ }^{\circ} \mathrm{C}$ (acetone). ${ }^{1} \mathrm{H}$ NMR (DMSO-d ${ }_{6}$ ): (Pyrazole) $\delta 8.28(1 \mathrm{H}, \mathrm{s}$, $\mathrm{OH}) ; 3.90(1 \mathrm{H}, \mathrm{d}, J 18.8, \mathrm{H} 4 \mathrm{a}) ; 3.53$ (1H, d, $J$ 18.8, H4b). (Thenoyl) $\delta 8.11(1 \mathrm{H}, \mathrm{dd}, J 3.8, J 1.4, \mathrm{H} 3) ; 7.98-7.97$ $(1 \mathrm{H}, \mathrm{m}, \mathrm{H} 5) ; 7.23(1 \mathrm{H}, \mathrm{dd}, J 4.8, J 4.0, \mathrm{H} 4)$. (Furyl) $\delta$ $7.98-7.97$ (1H, m, H5); 7.17 (1H, d, J 3.6, H3); 6.73 (1H, dd, J3.6, J 1.6, H4). ${ }^{13} \mathrm{C}$ NMR (DMSO-d $)$ ): (Pyrazole) $\delta 146.2(\mathrm{C} 3) ; 123.1\left(\mathrm{CF}_{3}, J_{\mathrm{CF}} 283.5\right) ; 91.9\left(\mathrm{C} 5,{ }^{2} J 34.5\right)$; 43.7 (C4). (Thenoyl) $\delta 158.7$ (C=O); 135.3 (C5); 135.2 (C3); 134.8 (C2); 127.1 (C4). (Furyl) $\delta 145.1$ (C2); 143.6 (C5); 115.1 (C4); 112.4 (C3). IR (KBr) $v_{\max } / \mathrm{cm}^{-1}: 3309$ $(\mathrm{OH}) ; 1620(\mathrm{C}=\mathrm{O}), 1512,1440(\mathrm{C}=\mathrm{C})$. Anal. Calc. for $\mathrm{C}_{13} \mathrm{H}_{9} \mathrm{~F}_{3} \mathrm{~N}_{2} \mathrm{O}_{3} \mathrm{~S}$ (330.28): C, 47.28; H, 2.75; N, 8.48\%. Found: C, 47.75; H, 2.76; N, 8.76\%.
Data for 3-(2-thienyl)-5-hydroxy-5-trifluoromethyl-4,5dihydro-1H-1-(2-thenoyl)pyrazole (5b)

Gray crystals (78\% yield), mp $155-157{ }^{\circ} \mathrm{C}$ (acetone). ${ }^{1} \mathrm{H}$ NMR (DMSO-d $)$ ): (Pyrazole) $\delta 8.29(1 \mathrm{H}, \mathrm{s}$, $\mathrm{OH}) ; 4.00$ (1H, d, J 18.8, H4a); 3.64 (1H, d, J 19.2, H4b). (Thenoyl) $\delta 8.08(1 \mathrm{H}, \mathrm{dd}, J 4.2, J 1.4, \mathrm{H} 3) ; 7.99(1 \mathrm{H}, \mathrm{dd}$, $J$ 4.8, $J$ 1.2, H5); $7.25-7.21$ (1H, m, H4). (Thienyl) $\delta$ $7.81(1 \mathrm{H}, \mathrm{d}, J$ 5.2, H5); $7.62(1 \mathrm{H}, \mathrm{d}, J 4.0, \mathrm{H} 3) ; 7.25-$ 7.21 (m, H4). ${ }^{13} \mathrm{C}$ NMR (DMSO-d 6 ): (Pyrazole) $\delta 148.2$ (C3); $123.1\left(\mathrm{CF}_{3}, J_{\mathrm{CF}} 284.6\right) ; 92.4\left(\mathrm{C} 5,{ }^{2} J 33.8\right) ; 44.5$ (C4). (Thenoyl) $\delta 158.4(\mathrm{C}=\mathrm{O}) ; 135.4$ (C5); 135.3 (C3); 134.6 (C2); 127.0 (C4). (Thienyl) $\delta 133.0$ (C2); 131.3 (C3); 130.5 (C5); 128.3 (C4). IR (KBr) $v_{\max } / \mathrm{cm}^{-1}: 3298$ $(\mathrm{OH}) ; 1619(\mathrm{C}=\mathrm{O}) ; 1510,1450(\mathrm{C}=\mathrm{C})$. Anal. Calc. for $\mathrm{C}_{13} \mathrm{H}_{9} \mathrm{~F}_{3} \mathrm{~N}_{2} \mathrm{O}_{2} \mathrm{~S}_{2}$ (346.34): C, 45.08; H, 2.62; N, 8.09\%. Found: C, 45.56; H, 2,60; N, 8.26\%.

Data for 3-(2-furyl)-5-hydroxy-5-trichloromethyl-4,5dihydro-1H-1-(2-thenoyl)pyrazole (6a)

Gray crystals (66\% yield), mp $125-127^{\circ} \mathrm{C}$ (acetone). ${ }^{1} \mathrm{H}$ NMR (DMSO-d ${ }_{6}$ ): (Pyrazole) $\delta 8.24$ (1H, s, OH); 4.00 (1H, d, $J$ 19.2, H4a); 3.76 (1H, d, $J$ 19.2, H4b). (Thenoyl) $\delta$ 8.09 (1H, dd, $J$ 3.8, $J$ 1.4, H3); $7.99-7.97(1 \mathrm{H}, \mathrm{m}, \mathrm{H} 5)$; $7.22(1 \mathrm{H}, \mathrm{m}, \mathrm{H} 4)$. (Furyl) $\delta 7.99-7.97(1 \mathrm{H}, \mathrm{m}, \mathrm{H}$ ) ; 7.22 $(1 \mathrm{H}, \mathrm{m}, \mathrm{H} 3) ; 6.73(1 \mathrm{H}, \mathrm{dd}, J 3.6, J 2.0, \mathrm{H} 4) .{ }^{13} \mathrm{C} \mathrm{NMR}$ (DMSO-d $)_{6}$ : (Pyrazole) $\delta 146.3(\mathrm{C} 3) ; 103.1\left(\mathrm{CCl}_{3}\right) ; 102.4$ (C5); 46.2 (C4). (Thenoyl) $\delta 160.6(\mathrm{C}=\mathrm{O}) ; 135.4$ (C5); 135.3 (C3); 134.8 (C2); 127.0 (C4). (Furyl) $\delta 145.2$ (C2); 144.7 (C5); 115.5 (C4); 112.4 (C3). IR (KBr) $v_{\max } / \mathrm{cm}^{-1}$ : $3329(\mathrm{OH}) ; 1634(\mathrm{C}=\mathrm{O})$; 1512, $1487(\mathrm{C}=\mathrm{C})$. Anal. Calc. for $\mathrm{C}_{13} \mathrm{H}_{9} \mathrm{Cl}_{3} \mathrm{~N}_{2} \mathrm{O}_{3} \mathrm{~S}$ (379.65): C, 41.13; H, 2.39; N, 7.38\%. Found: C, 41.46; H, 2.39; N, 7.19\%.

Data for 3-(2-thienyl)-5-hydroxy-5-trichloromethyl4,5dihydro-1H-1-(2-thenoyl)pyrazole (6b)

Gray crystals (62\% yield), mp $160-162{ }^{\circ} \mathrm{C}$ (acetone). ${ }^{1} \mathrm{H}$ NMR (DMSO-d ${ }_{6}$ ): (Pyrazole) $\delta 8.25(1 \mathrm{H}, \mathrm{s}, \mathrm{OH}) ; 4.12$ (1H, d, $J$ 19.2, H4a); 3.85 (1H, d, $J$ 19.2, H4b). (Thenoyl) $\delta$ $8.07(1 \mathrm{H}, \mathrm{dd}, J 3.8, J 1.0, \mathrm{H} 3) ; 8.00(1 \mathrm{H}, \mathrm{dd}, J 5.0, J 1.0$, H5); $7.24-7.21(1 \mathrm{H}, \mathrm{m}, \mathrm{H} 4)$. (Thienyl) $\delta 7.83(1 \mathrm{H}, \mathrm{d}, J 4.8$, $\mathrm{H} 5) ; 7.69$ (1H, d, J 3.2, H3); $7.24-7.21(1 \mathrm{H}, \mathrm{m}, \mathrm{H} 4) .{ }^{13} \mathrm{C}$ NMR (DMSO-d $)$ ): (Pyrazole) $\delta 149.9(\mathrm{C} 3) ; 103.0\left(\mathrm{CCl}_{3}\right)$; 102.9 (C5); 47.1 (C4). (Thenoyl) $\delta 160.2(\mathrm{C}=\mathrm{O}) ; 135.6$ (C5); 135.4 (C3); 134.5 (C2); 126.9 (C4). (Thienyl) $\delta 132.7$ (C2); 131.5 (C3); 130.7 (C5); 128.2 (C4). IR (KBr) $v_{\max } /$ $\mathrm{cm}^{-1}$ : $3165(\mathrm{OH}) ; 1605(\mathrm{C}=\mathrm{O}) ; 1509,1419(\mathrm{C}=\mathrm{C})$. Anal. Calc. for $\mathrm{C}_{13} \mathrm{H}_{9} \mathrm{Cl}_{3} \mathrm{~N}_{2} \mathrm{O}_{2} \mathrm{~S}_{2}$ (395.71): C, 39.46; H, 2.29; N, 7.08\%. Found: C, 39.30; H, 2.00; N, 6.69\%. 
Data for 3-(2-furyl)-5-hydroxy-5-trifluoromethyl-4,5dihydro-1H-1-(isonicotinoyl)pyrazole (7a)

Gray crystals (66\% yield), mp $164-166^{\circ} \mathrm{C}$ (acetone). ${ }^{1} \mathrm{H}$ NMR (DMSO-d $)$ : (Pyrazole) $\delta 8.48(1 \mathrm{H}, \mathrm{s}, \mathrm{OH}) ; 3.91$ (1H, d, J 19.2, H4a); 3.54 (1H, d, J 19.0, H4b). (Isonicotinoyl) $\delta 8.74(1 \mathrm{H}, \mathrm{s}, 2 \mathrm{H}, \mathrm{Py}) ; 7.59(2 \mathrm{H}, \mathrm{d}, J$ 5.6, Py). (Furyl) $\delta 7.87$ (1H, s, H5); 7.10 (1H, d, J3.6, H3); 6.67 (1H, dd, J3.4, J 1.8, H4). ${ }^{13} \mathrm{C}$ NMR (DMSO-d 6 ): (Pyrazole) $\delta 146.2$ (C3); $123.2\left(\mathrm{CF}_{3}, J_{\mathrm{CF}} 283.9\right)$; 91.7 (C5, ${ }^{2} J$ 33.7); 44.0 (C4). (Isonicotinoyl) $\delta 165.3$ (C:O); 149.8 (2C, Py); 142.7 (1C, Py); 122.6 (2C, Py). (Furyl) $\delta 144.9$ (C2); 144.5 (C5); 119.0 (C4); 115.5 (C3). IR (KBr) $v_{\max } / \mathrm{cm}^{-1}: 3153$ $(\mathrm{OH}) ; 1660(\mathrm{C}=\mathrm{O}) ; 1546,1494(\mathrm{C}=\mathrm{C})$. Anal. Calc. for $\mathrm{C}_{14} \mathrm{H}_{10} \mathrm{~F}_{3} \mathrm{~N}_{3} \mathrm{O}_{3}$ (325.25): C, 51.70; H, 3.10; N, 12.92\%. Found: C, 51.94; H, 3.03; N, 12.88\%.

Data for 3-(2-thienyl)-5-hydroxy-5-trifluoromethyl-4,5dihydro-1H-1-(isonicotinoyl)pyrazole (7b)

Gray crystals (50\% yield), mp $156-158{ }^{\circ} \mathrm{C}$ (acetone). ${ }^{1} \mathrm{H}$ NMR (DMSO-d $)$ ): (Pyrazole) $\delta 8.49(1 \mathrm{H}, \mathrm{s}, \mathrm{OH}) ; 4.03$ (1H, d, J 19.0, H4a); 3.66 (1H, d, J 19.2, H4b). (Isonicotinoyl) $\delta 8.75$ (2H, s, Py); $7.62-7.58(2 \mathrm{H}, \mathrm{m}, \mathrm{Py})$. (Thienyl) $\delta 7.75(1 \mathrm{H}, \mathrm{dd}, J 5.2, J 1.2, \mathrm{H} 5) ; 7.62-7.58(1 \mathrm{H}$, m, H3); 7.18 (1H, dd, J 5.1, J 3.7, H4). ${ }^{13} \mathrm{C}$ NMR (DMSO$\left.\mathrm{d}_{6}\right)$ : (Pyrazole) $\delta 149.0$ (C3); $123.1\left(\mathrm{CF}_{3}, J_{\mathrm{CF}} 283.7\right) ; 92.2$ (C5, ${ }^{2} J$ 33.6); 44.8 (C4). (Isonicotinoyl) $\delta 164.8$ (C:O); 149.7 (2C, Py); 142.4 (1C, Py); 122.7 (2C, Py). (Thienyl) $\delta$ 132.8 (C2); 131.5 (C3); 130.4 (C5); 128.2 (C4). IR (KBr) $v_{\text {max }} / \mathrm{cm}^{-1}: 3078(\mathrm{OH}) ; 1652(\mathrm{C}=\mathrm{O}) ; 1551,1446(\mathrm{C}=\mathrm{C})$. Anal. Calc. for $\mathrm{C}_{14} \mathrm{H}_{10} \mathrm{~F}_{3} \mathrm{~N}_{3} \mathrm{O}_{2} \mathrm{~S}$ (341.31): C, 49.27; H, 2.95; N, $12.31 \%$. Found: C, 49.28; H, 3.17; N, 12.45\%.

Data for 3-(2-furyl)-5-hydroxy-5-trichloromethyl-4,5dihydro-1H-1-(isonicotinoyl)pyrazole $(\mathbf{8 a})$

Gray crystals (65\% yield), mp $155-157^{\circ} \mathrm{C}$ (acetone). ${ }^{1} \mathrm{H}$ NMR (DMSO-d $)$ ): (Pyrazole) $\delta 8.41(1 \mathrm{H}, \mathrm{s}, \mathrm{OH}) ; 4.01$ (1H, d, J $19.2 \mathrm{H} 4 \mathrm{a}$, ); 3.72 (1H, d, J 19.4, H4b). (Isonicotinoyl) $\delta 8.75$ (2H, d, J 4.2, Py); 7.59 (2H, d, J 6.0, Py). (Furyl) $\delta 7.84$ (1H, s, H5); 7.13 (1H, d, J 3.4, H3); 6.65 (1H, dd, J 3.4, J 1.8, H4). ${ }^{13} \mathrm{C} \mathrm{NMR}$ (DMSO-d 6 ): (Pyrazole) $\delta 146.0$ (C3); $103.0\left(\mathrm{CCl}_{3}\right) ; 101.8$ (C5); 46.6 (C4). (Isonicotinoyl) $\delta 166.7$ (C:O); 149.3 (2C, Py); 143.3 (1C, Py); 122.4 (2C, Py). (Furyl) $\delta 145.2$ (C2); 144.5 (C5); 115.4 (C4); 112.1 (C3). IR (KBr) $v_{\text {max }} / \mathrm{cm}^{-1}: 3288(\mathrm{OH}) ; 1654$ $(\mathrm{C}=\mathrm{O}) ; 1554,1482(\mathrm{C}=\mathrm{C})$. Anal. Calc. for $\mathrm{C}_{14} \mathrm{H}_{10} \mathrm{Cl}_{3} \mathrm{~N}_{3} \mathrm{O}_{3}$ (374,61): C, 44.89; H, 2.69; N, 11.22\%. Found: C, 44.59; $\mathrm{H}, 2.55 ; \mathrm{N}, 11.05 \%$.
Data for 3-(2-thienyl)-5-hydroxy-5-trichloromethyl-4,5dihydro-1H-1-(isonicotinoyl)pyrazole (8b)

Gray crystals (60\% yield), mp $169-171{ }^{\circ} \mathrm{C}$ (acetone). ${ }^{1} \mathrm{H}$ NMR (DMSO-d $)$ ): (Pyrazole) $\delta 8.49$ (1H, s, OH); 4.11 (1H, d, J 19.4, H4a); 3.81 (1H, d, J 19.4, H4b). (Isonicotinoyl) $\delta 8.74(2 \mathrm{H}, \mathrm{d}, J 5.8, \mathrm{Py}) ; 7.62-7.56(2 \mathrm{H}$, m, Py). (Thienyl) $\delta 7.72$ (1H, d, J 5.0, H5); $7.62-7.56(1 \mathrm{H}$, $\mathrm{m}, \mathrm{H} 3) ; 7.16$ (1H, dd, $J$ 5.0, J 3.6, H4). ${ }^{13} \mathrm{C} \mathrm{NMR}$ (DMSO$\mathrm{d}_{6}$ ): (Pyrazole) $\delta 149.0$ (C3); $102.9\left(\mathrm{CCl}_{3}\right) ; 102.5$ (C5); 47.3 (C4). (Isonicotinoyl) $\delta 165.4(\mathrm{C}: \mathrm{O}) ; 150.1$ (2C, Py); 142.4 (1C, Py); 122.7 (2C, Py). (Thienyl) $\delta 132.5$ (C2); 131.4 (C3); 130.4 (C5); 128.1 (C4). IR (KBr) $v_{\max } / \mathrm{cm}^{-1}$ : $3231(\mathrm{OH}) ; 1634(\mathrm{C}=\mathrm{O}) ; 1597,1444(\mathrm{C}=\mathrm{C})$. Anal. Calc. for $\mathrm{C}_{14} \mathrm{H}_{10} \mathrm{Cl}_{3} \mathrm{~N}_{3} \mathrm{O}_{2} \mathrm{~S}$ (390.67): C, 43.04; H, 2.58; N, $10.76 \%$. Found: C, 43.33; H, 2.62; N, 10.92\%.

\section{Acknowledgments}

The authors are thankful to Conselho Nacional de Desenvolvimento Científico e Tecnológico - CNPq (Process No. 303636/2002-5) for financial support. Fellowships from Coordenação de Aperfeiçoamento de Pessoal de Nível Superior - CAPES ( to M. R. Oliveira and M. B. Costa and L. B. Silva ) are also acknowledged.

\section{References}

1. Katritzky, A.R.; Rees, C.W.; Scriven, E. F.; Comprehensive Heterocyclic Chemistry II , Pergamon Press: Oxford, 1996, vol. 3.

2. Zelenin, K. N.; Org. Prep. Proc. Int. 1995, 27, 519.

3. Singh, S. P.; Kumar, D.; Batra, H.; Naithani, R.; Rozas, I.; Elguero J.; Can. J. Chem. 2000, 78, 1109.

4. Taylor, E. C.; Patel, H. H.; Kumar, H.; Tetrahedron 1992, 48, 8089.

5. Roelfvan, S. G.; Arnold, C.; Wellnga, K.; J. Agric. Food Chem. 1979, 84, 406.

6. Kedar, R. M.; Vidhale, N. N.; Chincholkar, M. M.; Orient. J. Chem. 1997, 13, 143.

7. Singh, A.; Rathod, S.; Berad, B. N.; Patil, S. D.; Dosh, A. G.; Orient. J. Chem. 2000, 16, 315.

8. Katri, H. Z.; Vunii, S. A.; J. Ind. Chem. Soc. 1981, 58, 1968.

9. Das, N. B.; Mittra, A. S.; Ind. J. Chem. 1978, 16B, 638.

10. Azarifar, D.; Shaebanzadeh, M.; Molecules 2002, 7, 885.

11. Holla, B. S.; Akaberali, P. M.; Shivanada, M. K.; Farmaco 2000, 55, 256.

12. Palaska, E.; Aytemir, M.; Tayfun, I.; Erol, K.; Dilek, E.; Eur. J. Med. Chem. Chim. Ther. 2001, 36, 539.

13. Regaila, H. A.; El-Bayonk, A. K.; Hammad, M.; Egypt. J. Chem. 1979, 20, 197. 
14. Krishna, R.; Pande, B. R.; Bharthwal, S. P.; Parmar, S. S.; Eur. J. Med. Chem. 1980, 15, 567.

15. Husain, M. I.; Shukla, S.; Ind. J. Chem. 1986, 25B, 983.

16. Mamolo, M. G.; Zampieri, D.; Falagiani, V.; Vio, L.; Banfi, E.; Il Farmaco 2001, 56, 593.

17. Flores, A. F. C.; Brondani, S.; Zanatta, N.; Rosa, Adriano; Martins, M. A. P.; Tetrahedron Lett. 2002, 43, 8701.

18. Bonacorso, H. G.; Lang, E. S.; Lewandowski, H.; Martins, M. A. P.; Peppe,C.; Zanatta, N.; Inorg. Chem. Commun. 2003, 6, 646; Martins, M. A. P.; Pereira,C. M. P.; Zimmermann, N. E. K.; Moura, S.; Sinhorin, A. P.; Cunico, W.; Zanatta, N.; Bonacorso, H. G.; Flores, A. C. F.; Synthesis 2003, 2353; Martins, M. A. P.; Bastos, G. P.; Sinhorin, A. P.; Zimmermann, N. E. K.; Rosa, A.; Brondani, S.; Emmerich, D.; Bonacorso, H. G.; Zanatta, N.; J. Fluorine Chem. 2003, 123, 249; Martins, M. A. P.; Pereira, C. M. P.; Zimmermann, N. E. K.; Cunico,W.; Moura, S.; Beck, P.; Zanatta, N.; Bonacorso, H. G.; J. Fluorine Chem. 2003, 123, 261; Martins, M. A. P.; Pereira, C. M. P.; Beck, P.; Machado, P.; Moura, S.; Teixeira, M. V. M.; Bonacorso, H. G.; Zanatta, N.; Tetrahedron Lett. 2003, 44, 6669; Zanatta, N.; Barichello, R.; Pauletto, M. M.; Bonacorso, H. G.; Martins, M. A. P.; Tetrahedron Lett. 2003, 44, 961; Zanatta, N.; Rosa, L. S.; Cortelini, M. F. M.; Beux, S.; Santos, A. P. D.; Bonacorso, H. G.; Martins, M. A. P.; Synthesis 2002, 2404; Martins, M. A. P.; Flores, A. F. C.; Bastos, G. P.; Sinhorin, A. P.; Bonacorso, H. G.; Zanatta, N.; Tetrahedron Lett. 2000, 41, 293.
19. Bonacorso, H. G.; Lopes, I. S.; Wastowski, A. D.; Zanatta, N.; Martins, M. A. P.; J. Fluorine Chem. 2003, 120, 29; Zanatta, N.; Madruga, C. C.; Marisco, P. C.; Flores, D. C.; Bonacorso, H. G.; Martins, M. A. P.; J. Heterocycl. Chem. 2000, 37, 1213; Zanatta, N.; Cortelini, M. F.; Carpes, M. J. S.; Bonacorso, H. G.; Bittencourt, S. R. T.; Lourega, R. V.; Flores, A. F. C.; Zanatta, N.; Martins, M. A. P.; Synthesis 2000, 1431; Bonacorso, H. G.; Duarte, S. H. G.; Zanatta, N.; Martins, M. A. P.; Synthesis 2002, 1037.

20. Bonacorso, H. G.; Bittencourt, S. T.; Wastowski, A. D.; Wentz, A. P.; Zanatta, N.; Martins, M. A. P.; Tetrahedron Lett. 1996, 37, 9155; Bonacorso, H. G.; Marques, L. L. M.; Zanatta, N.; Martins, M. A. P.; Synth. Commun. 2002, 32, 3225.

21. Bonacorso, H. G.; Muniz, M. N.; Wastowski, A. D.; Zanatta, N.; Martins, M. A. P.; Heteroatom Chem. 2003, 14, 132; Zanatta, N.; Flores, D. C.; Madruga, C. C.; Faoro, D.; Flores, A. F. C.; Bonacorso, H. G.; Martins M. A. P.; Synthesis 2003, 894; Bonacorso, H. G.; Lourega, R. V.; Wastowski, A. D.; Flores, A. F. C.; Zanatta, N.; Martins, M. A. P.; Tetrahedron Lett. 2002, 43, 9315; Bonacorso, H. G.; Wentz, A. P.; Zanatta, N.; Martins, M. A. P.; Synthesis 2001, 1505; Bonacorso, H. G.; Wastowski, A. D.; Muniz, M. N.; Zanatta, N.; Martins, M. A. P.; Synthesis 2002, 1079; Flores, A. F. C.; Brondani, S; Zanatta, N.; Rosa, A.; Martins, M. A. P.; Tetrahedron Lett. 2003, 43, 8701 . 